\title{
Decreased Motivation Following Cocaine Self-Administration Under Extended Access Conditions: Effects of Sex and Ovarian Hormones
}

\author{
Wendy J Lynch*,' and Jane R Taylor' \\ 'Department of Psychiatry, Yale University, New Haven, CT, USA
}

\begin{abstract}
We have previously shown that following extended access to cocaine, females, but not males, show marked increases in motivation to obtain cocaine, and we have hypothesized that such changes may contribute to long-term compulsive aspects of addiction that result in a persistent vulnerability to relapse. Here, we investigate the effects of extended cocaine access on short-term motivational changes in both male and female rats. An additional goal was to determine whether estrogen modulates motivation to self-administer cocaine in female rats following extended access to cocaine. A discrete trial procedure was used that allowed rats $24-\mathrm{h}$ access to cocaine infusions ( $1.5 \mathrm{mg} /$ $\mathrm{kg}$ ) that were available in discrete trials (four, 10-min trials/h) for 7 consecutive days. Motivation to obtain cocaine was assessed by responding under a progressive ratio schedule, and preference for sucrose (1\%) vs water was assessed using a two-bottle, 24-h test. Each was assessed prior to and immediately following discrete trial cocaine self-administration. Results showed that following discrete trial cocaine self-administration, both males and females showed a decrease in preference for sucrose, but only females showed a reduction in levels of responding for cocaine under the progressive ratio schedule, suggesting a sex difference in motivation that was specific to cocaine. Subsequent studies with ovariectomized rats showed that estrogen replacement blocked the decrease in motivation to obtain cocaine infusions. Together, these findings suggest that there is a dissociation between sex and the short- vs long-term alteration in motivation to use cocaine. The mechanisms that may underlie these observed sex differences, including the role of estrogen, are discussed.
\end{abstract}

Neuropsychopharmacology (2005) 30, 927-935, advance online publication, 12 January 2005; doi: I 0.1 038/sj.npp. 1300656

Keywords: sex differences; cocaine; self-administration; motivation; sucrose; withdrawal

\section{INTRODUCTION}

Cocaine addiction in humans is characterized by periods of heavy use, or binges, followed by periods of abstinence in which craving and motivation to use the drug progressively increase (Gawin and Kleber, 1986; Jentsch and Taylor, 1999). During active binge cycles, addicts report increasing difficulty in abstaining from cocaine use, despite the continually decreasing hedonic effects of cocaine with repeated use (Robinson and Berridge, 2001). During abstinence periods, motivation to use cocaine appears to be low during the initial withdrawal period (or the 'crash'), but to progressively increase over time. The initial withdrawal period is also associated with affective disturbances

\footnotetext{
*Correspondence: Dr WJ Lynch, Department of Psychiatry, Yale University, New Haven, CT 06508, USA, Tel: + I 2039371925, Fax: + I 2039747897, E-mail: wendy.lynch@yale.edu

Received 24 June 2004; revised 8 November 2004; accepted 15 November 2004

Online publication: 19 November 2004 at http://www.acnp.org/citations/ NPPI | | 90404029 |/default.pdf
}

including lethargy, dysphoria, and anhedonia (eg American Psychiatric Association, 1994; Gawin and Kleber, 1986; Mulvaney et al, 1999; Kampman et al, 2002). These symptoms have been reported to develop within hours of abstinence and may persist weeks or even months (Gawin and Kleber, 1986).

A similar time course and pattern of motivational changes have been observed in laboratory animals following chronic cocaine exposure. For example, recent work with male rats has revealed that levels of cue-induced reinstatement responding (Grimm et al, 2001) and motivation to obtain cocaine, as measured by responding under a progressive ratio schedule (Morgan et al, 2002), are low during initial abstinence periods, but that they progressively increase over time presumably due to dissipation of tolerance (Koob et al, 2004; Ahmed et al, 2002) and/or to the development of sensitization to the reinforcing effects of cocaine (Robinson and Berridge, 2001). Additionally, like humans, laboratory animals seem to display a reduced hedonic capacity (or anhedonia) during cocaine withdrawal. Anhedonia has been defined as reduced interest in or pleasure from normally rewarding stimuli (Markou and Koob, 1991) and is one of 
the prominent symptoms of both depression and cocaine withdrawal (American Psychiatric Association, 1994). In laboratory animals, this phenomenon is most commonly demonstrated by a reduction in responding for brain selfstimulation reward (Markou and Koob, 1991; Kokkinidis and McCarter, 1990), but has also been demonstrated by a reduction in preference for nondrug rewards (Barr and Phillips, 1999, 2002). For example, a preference for sucrose (ie $1-10 \%$ ) and other sweet solutions over water is usually found in a free choice self-administration procedure, and during stimulant withdrawal, this preference decreases (Barr and Phillips, 2002). It is assumed that the decrease in preference represents a change in the animal's ability to experience pleasure (ie anhedonia).

Although numerous animal studies have investigated motivational and behavioral changes that occur following chronic cocaine exposure, most of these studies have examined the behavior of male laboratory animals, and to date, none have investigated sex differences in the effects of cocaine on short-term motivational changes. We have recently found sex differences in the effects of chronic, extended access cocaine self-administration on long-term motivational changes (Lynch and Taylor, 2004). Specifically, following 24-h/day access to cocaine infusions that were available under a discrete trial procedure (10 min trials, four trials/h, 7 days), females, but not males, showed a marked increase in motivation to obtain cocaine infusions after a 10-day abstinence period (as assessed by responding under a progressive ratio schedule). Such alterations in motivation following extended access exposure may be critical for longterm compulsive aspects of addiction that result in a persistent vulnerability to relapse. Here, we investigate the effect of high levels of cocaine self-administration on shortterm motivational changes in both male and female rats that may be involved in hedonic/motivational aspects of cocaine addiction. Motivation to obtain cocaine infusions was assessed prior to and immediately following extended access cocaine self-administration using the progressive ratio schedule. With this procedure, the ratio requirement to obtain an infusion of drug progressively increases within a session and the final ratio completed is believed to be a sensitive measure of motivation (Richardson and Roberts, 1996). Preference for a nondrug reward (sucrose) was also measured in separate groups of rats during a similar time frame as the progressive ratio self-administration testing period using a 24 -h two-bottle test.

A second goal of this study was to investigate the biological basis of sex differences in motivation to obtain cocaine. Previous research has revealed that ovarian hormones play a critical role in modulating the reinforcing effects of cocaine in both women (Sofuoglu et al, 1999) and female laboratory animals (for reviews see Carroll et al, 2004; Lynch et al, 2001; Becker, 1999). For example, studies with laboratory animals have shown that female rats responding for cocaine under a progressive ratio schedule reach break points considerably higher than those observed in male rats, particularly when females are in the estrous phase of the estrous cycle (Roberts et al, 1989). Results obtained from ovariectomized (OVX) female rats suggest that estrogen, in particular, may play an important role in modulating the reinforcing effects of cocaine. For example, previous work has shown that female rats acquire cocaine self-administration at a faster rate than do male rats, and that OVX blocks this sex difference, whereas estrogen replacement restores it ( $\mathrm{Hu}$ et al, 2004; Lynch and Carroll, 1999; Carroll et al, 2002). Few studies, however, have investigated the role of estrogen under extended cocaine access conditions. Thus, in the present experiments, we examined the role of estrogen replacement in OVX female rats on levels of cocaine self-administration under extended access conditions and on subsequent motivation to obtain cocaine.

\section{METHODS}

\section{Subjects}

Subjects were age-matched, sexually mature male $(n=6)$ and intact female $(n=8)$ and OVX female $(n=14)$ SpragueDawley rats between 90 and 100 days. Rats weighed between 280-320 g (female) and 380-430 g (male) at the beginning of the study. Rats were housed individually in the colony room and were maintained on a 12-h light/dark cycle (lights on at 0700). Rats had free access to food and water throughout the study. After a minimum of 5 days from arrival at the facility, each rat was implanted with a chronic indwelling cannula into the right jugular vein using methods that have been described previously (Lynch et al, 2000). A separate group of male $(n=6)$ and intact female $(n=5)$ rats were given access to water and a $1 \%$ sucrose solution, and prior to cannulation and self-administration training, these rats were prescreened for sucrose preference as described below. After cannulation, rats were placed individually in operant test chambers (ENV-018M; Med Associates, St Albans, VT), and once grooming and eating behaviors resumed, behavioral testing began (typically after $24 \mathrm{~h}$ of recovery). The experimental protocol was approved by the Animal Care and Use Committee of Yale University.

\section{Drugs}

Cocaine hydrochloride was purchased from Sigma Chemical Company (St Louis, MO). Cocaine was mixed in sterile $0.9 \%$ saline and passed through a microfilter. Cocaine infusions $(1.5 \mathrm{mg} / \mathrm{kg})$ were delivered at a rate of $0.024 \mathrm{ml} / \mathrm{s}$ and the infusion duration was adjusted according to the body weight of each subject $(1.5 \mathrm{~s} / 100 \mathrm{~g})$. A relatively high dose $(1.5 \mathrm{mg} / \mathrm{kg} /$ infusion) of cocaine was selected based on previous findings from our laboratory showing that this dose maintains similar levels of responding under the progressive ratio schedule in males and females prior to 24$\mathrm{h}$ access to cocaine under the discrete trial procedure (Lynch and Taylor, 2004), and maintained high levels of intake under the discrete trial procedure in both males and females (Lynch and Taylor, 2004; Lynch et al, 2004). In all, $17 \beta$-estradiol benzoate (EB) was purchased from SigmaAldrich (St Louis, MO) and dissolved in peanut oil $(0.5 \mathrm{mg} /$ $\mathrm{ml})$. The dose of EB selected $(0.5 \mathrm{mg} / \mathrm{kg})$ was based on previous work showing that this dose reinstates sexual receptivity in OVX rats (Pfaus and Pfaff, 1992) and previous work from our group showing that this dose produces rapid rates of acquisition of cocaine self-administration in OVX rats (Lynch et al, 2001). 


\section{Experimental Procedures}

Cocaine self-administration training under a fixed ratio schedule. Cocaine self-administration training began a minimum of 1 day after surgery. Daily sessions began at 1200 , and rats had access to cocaine (1.5 mg/kg/infusion) under a fixed ratio one schedule. Each session began with the introduction of the left lever (cocaine associated) into the operant chamber. Each response on this lever produced an infusion of cocaine, and the stimulus light above the lever was illuminated for the duration of the infusion (approximately $5 \mathrm{~s}$ ). The left lever remained extended into the chamber and cocaine infusions were available until a total of 20 infusions were obtained. Each rat received one noncontingent, experimenter delivered intravenous infusion of cocaine at the beginning of each training session. The right lever ('activity lever') was extended into the chamber throughout experiment, and responses on it were recorded but produced no consequence. Responding was assessed daily under the fixed ratio schedule until rats met the criterion for acquisition. Acquisition of cocaine selfadministration was defined as five consecutive sessions in which rats earned all 20 infusions that were available.

Cocaine self-administration under a progressive ratio schedule. Once rats acquired cocaine self-administration under a fixed ratio schedule, responding was assessed for three consecutive sessions under a progressive ratio schedule. At the beginning of each progressive ratio session, the left lever (cocaine-associated lever) was extended in to operant chamber and remained extended until 10-min before the next progressive ratio session. Each rat received one noncontingent, experimenter delivered intravenous infusion of cocaine at the beginning of each session. Responding on the left lever was reinforced with cocaine $(1.5 \mathrm{mg} / \mathrm{kg} /$ infusion$)$ under a progressive ratio schedule in which the ratio requirement increased progressively throughout each daily session in the following steps: 1,2 , $4,6,9,12,15,20,25,32,40,50,62,77,95,118,145,178,219$, $268,328,402,492,603$, etc (procedure described in detail in Richardson and Roberts, 1996). The right lever ('activity lever') was extended into the chamber for the duration of the experiment, and responses on it were recorded but were without consequence.

Cocaine self-administration under a discrete trial procedure. The discrete trial procedure used has been described previously (Fitch and Roberts, 1993; Roberts et al, 2002; Lynch and Taylor, 2004; Lynch et al, 2004; Morgan et al, 2002). Briefly, after the third progressive ratio session, cocaine self-administration under a discrete trial procedure was assessed for 7 consecutive days. Trials were initiated every $15 \mathrm{~min}$ and each 10 -min trial began with the introduction of the left lever into the chamber. A response made on the left lever during a 10-min trial produced an infusion of cocaine $(1.5 \mathrm{mg} / \mathrm{kg} /$ infusion), and the stimulus light above the lever was illuminated for the duration of the infusion (approximately $5 \mathrm{~s}$ ). A discrete trial was terminated and the lever was retracted following a response or after $10 \mathrm{~min}$ had elapsed. The right lever ('activity lever') was extended into the chamber for the duration of the experiment, and responses on it were recorded but produced no consequence. Following discrete trial cocaine self-administration, rats were either retested for sucrose preference or for cocaine self-administration under the progressive ratio schedule.

Sucrose preference. A separate group of the rats tested on discrete trial cocaine self-administration were tested on preference for sucrose prior to cocaine self-administration training and immediately after the last discrete trial cocaine self-administration session ( $n=6$ males and 5 females). For these rats, testing for sucrose preference began a minimum of $48 \mathrm{~h}$ after arrival at the facility based on methods that have been described previously (Stock et al, 2000). Briefly, rats were given access to a $1 \%$ sucrose solution in addition to the tap water that was already available. Both bottles were weighed daily to record the amount of sucrose and water consumed. Water and sucrose solutions were changed every other day, and the presentation side (left $v s$ right) was alternated daily to control for side preferences. Rats had ad libitum access to both water and sucrose for an average of 10 days. Preference for sucrose over water was calculated for the last four sessions under the two-bottle test, and it was defined as a mean of $60 \%$ or greater. Only rats showing a preference for sucrose were used in the self-administration studies (approximately $80 \%$ ). In order to determine changes in preference for sucrose during withdrawal from cocaine, rats were retested on intake of sucrose and water immediately following the last discrete trial cocaine selfadministration session. Intake of sucrose and water was recorded for 4 consecutive days. The conditions at retest were identical to those used initially.

Progressive ratio responding for cocaine following discrete trial self-administration. After the last cocaine selfadministration session under the discrete trial procedure, cocaine self-administration under a progressive ratio schedule was reassessed in the group of male and intact female rats that were not prescreened for sucrose preference and in two groups of OVX female rats. Beginning $10 \mathrm{~min}$ after the last discrete trial cocaine self-administration session, cocaine infusions were available under a fixed ratio one schedule for one session to insure cannula patency using conditions identical to those described above. Subsequently, cocaine was available under a progressive ratio schedule for three consecutive sessions under identical conditions as those used initially (see above). Thus, the first, second, and third progressive ratio retest session began 24,48 , and $72 \mathrm{~h}$ after the last discrete trial cocaine selfadministration session, respectively.

Estrogen replacement and vaginal cytology. In order to determine the effect of estrogen on levels of cocaine selfadministration under extended access conditions and on subsequent motivation to obtain cocaine, OVX rats with and without estrogen replacement were tested. This approach was taken since previous work (King et al, 1993) and unpublished results from our laboratory have shown that cocaine disrupts the estrous cycle in intact female rats making it difficult to draw conclusions regarding the effect of ovarian hormones on cocaine selfadministration. OVX rats were purchased from Charles 
River Laboratory and ovariectomies were performed no more than 1 week (or less than 4 days) prior to the arrival at the laboratory. OVX rats were randomly assigned to either an $\mathrm{EB}$ group $(\mathrm{OVX}+\mathrm{EB})$ or a vehicle group $(\mathrm{OVX}+\mathrm{VEH})$. Beginning 3 days after arrival at the facility, rats were given daily subcutaneous injections of $\mathrm{EB}(0.5 \mathrm{mg} / \mathrm{kg})$ or an equal volume of peanut oil at 1130 . Since estrogen has previously been shown to affect feeding behavior (Tarttelin and Gorski, 1973), self-administration sessions began a minimum of 7 days after the first injection to allow food intake to stabilize. In order to obtain information regarding hormonal status, OVX rats were vaginally swabbed daily at 1130 . Additionally, in order to determine whether the estrous cycle was functioning in intact females self-administering high levels of cocaine, several intact females rats were also vaginal swabbed and estrous cycle phase was determined. Although this was carried out randomly in only a few rats, and the data are not presented, results indicate that the estrous cycle was disrupted (as evidenced by fewer than predicted proestrus and estrous cycle phases). In order to control for the effect of handling, several male rats were randomly 'mock' swabbed during a similar time frame as the females. The testing procedure did not interfere with the experimental protocol. Vaginal swabs were placed on slides, stained with cresyl violet, coverslipped, and examined under light microscopy. Proestrus and estrous were characterized by the presence of predominantly nucleated epithelial cells and predominantly non-nucleated cornified epithelial cells, respectively. The metestrus/diestrus phase was characterized by the presence of leukocytes and necrotic epithelia.

\section{Data Analysis}

The data from the group of intact female and the group of male rats that were prescreened on sucrose preference were compared with the group of intact female and the group of male rats that were not prescreened on sucrose preference using unpaired $t$-tests. Since the two groups of females and the two groups of males did not differ on cocaine selfadministration under the fixed ratio, progressive ratio, or discrete trial schedules, the data from these groups were pooled and analyzed together $(n=13$ females, $n=12$ males). The mean number of days to acquire cocaine selfadministration observed under the fixed ratio schedule and mean number of infusions obtained under the initial three progressive ratio sessions was compared between males and intact females and between the OVX $+\mathrm{EB}$ and OVX $+\mathrm{VEH}$ groups using separate $t$-tests. The mean daily intake over the 7 days of access to cocaine under the 24-h access discrete trial procedure was compared between intact female and male rats and between the OVX + EB and the OVX + VEH groups using separate repeated measures ANOVA. The percent preference for sucrose was determined for intact female $(n=5)$ and male $(n=6)$ rats for each of the 4 days at baseline and compared to the 4 days at retest using a repeated measures ANOVA, with time and days as the within-subject factors and sex as the betweensubject factor. Final ratios reached and the mean number of infusions obtained at baseline and at retest were determined for the intact female $(n=8)$ and male $(n=6)$ rats that were retested on progressive ratio responding following discrete trial cocaine self-administration. The mean number of infusions was compared using a repeated measures ANOVA, with time (before $v s$ after) and day as the within-subject factors and sex as the between-subject factor. Since the progressive ratio data from one of the intact females was greater than 2 SDs above the mean, data from this animal were not included in the final analysis $(n=7$ females). All statistical analyses were conducted using SPSS 10 , and findings were considered statistically significant if $p$-values were less than 0.05 .

\section{RESULTS}

\section{Effect of Sex on Initial Cocaine Self-Administration Under the Fixed Ratio, Progressive Ratio, and Discrete Trial Sessions}

The mean number of days to acquire cocaine selfadministration under the fixed ratio schedule did not differ significantly between males $(10.3 \pm 1.0)$ and females $(9.4 \pm 0.9)(p>0.1)$. Additionally, under the three sessions in which cocaine was available under a progressive ratio schedule, males and females obtained a similar number of infusions ( $14.9 \pm 1.0$ and $13.9 \pm 1.2$, respectively) $(p>0.1)$, indicating that prior to extended access to cocaine, males and females were equally motivated to obtain infusions of cocaine. Sex differences emerged, however, under the 24-h access discrete trial procedure with females self-administering consistently more cocaine per day than did males (see Figure 1a). Patterns of intake over the 7-day testing period were similar in males and females and were characterized by maximal levels of intake during the first 1-2 days followed by a decrease and stabilization of intake at a lower level. As with previous work (Lynch and Taylor, 2004), male rats tended to show a greater decrease in cocaine intake following the initial days of access under these conditions (day 4). A repeated measures ANOVA revealed a significant effect of sex $\left(\mathrm{F}_{1,23}=4.5, p<0.05\right)$ and day $\left(\mathrm{F}_{6,138}=4.3, p<0.001\right)$, but no significant interaction of
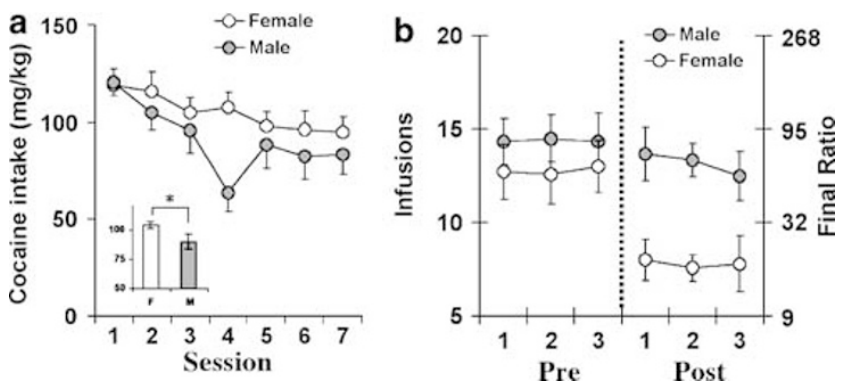

Figure I Effect of sex on responding under the discrete trial and progressive ratio cocaine self-administration procedures. (a) Data represent the mean $( \pm$ SE) daily cocaine intake for intact female $(n=13$, open circles) and male ( $n=12$, filled circles) rats as a function of day under the discrete trial procedure. The inset shows the mean $( \pm S E)$ daily intake over the 7 day testing period for males $(M)$ and females (F). Asterisks indicate a significant group difference $(p<0.05)$. (b) Mean $( \pm$ SE) number of infusions obtained and the corresponding final ratios reached under the progressive ratio schedule for the three baseline sessions before (Pre) and the three retest sessions after (Post) access to cocaine under the discrete trials procedure for male (filled circles, $n=6$ ) and female (open circles, $n=7$ ) rats. 
sex by day $(p>0.1)$. Responses on the activity lever were very low throughout the experiment, and there were no significant differences between males and females (data not shown).

\section{Effect of Sex on Progressive Ratio Responding for Cocaine Prior to and Following Extended Access Cocaine Self-Administration}

Figure $1 \mathrm{~b}$ shows data for the number of infusions and corresponding final ratios reached under the progressive ratio schedule for male and female rats before and after cocaine self-administration under the discrete trial procedure. We observed sex differences in motivational changes following cocaine self-administration under the discrete trial procedure. Specifically, final ratios reached under the three progressive ratio retest sessions were decreased from those observed at the three baseline sessions by a mean of $33 \%( \pm 7 \%)$ in females compared to only $5 \%( \pm 12 \%)$ in males. The decrease observed in females was a persistent baseline change and values were relatively constant across the three retest sessions. A repeated measures ANOVA revealed a significant main effect of time (Pre vs Post; $\left.\mathrm{F}_{1,11}=11.3, \quad p<0.01\right)$, sex $\left(\mathrm{F}_{1,11}=6.0, p<0.05\right)$, and $\mathrm{a}$ significant interaction of sex and time $\left(\mathrm{F}_{1,11}=5.0\right.$, $p<0.05)$, but no significant effect of day $(p>0.1)$. Further analysis within females and within males using separate repeated measures ANOVA revealed a significant effect of time for females $\left(\mathrm{F}_{1,6}=26.7, p<0.01\right)$, but not males $(p>0.1)$. Notably, a Pearson correlation analysis revealed that there was no relationship between average total intake over the 7-day discrete trial procedure and the percent change from baseline (average number of infusions obtained during the three initial progressive ratio sessions) to retest (average number of infusions obtained during the three retest progressive ratio sessions) for final ratios $\left(r^{2}=0.01\right.$ females, $r^{2}=0.07$ males), indicating that the sex difference in intake cannot account for the decreased breakpoints observed in females. Thus, following extended access cocaine self-administration, females, but not males, showed a marked decrease in motivation to obtain cocaine infusions.

\section{Effect of Sex on Sucrose Preference before and after Extended Access to Cocaine}

Figure 2 shows data on preference for sucrose over water prior to cocaine self-administration and after discrete trial self-administration. Prior to cocaine self-administration training, sucrose preference was comparable between males and females and averaged $70( \pm 3 \%)$ and $67( \pm 4 \%)$, respectively. At retest, sucrose preference was decreased from that observed at baseline in both males and females and averaged $54( \pm 6 \%)$ and $59( \pm 7 \%)$, respectively. A repeated measures ANOVA revealed a significant effect for time (Pre $v s$ Post; $\mathrm{F}_{1,9}=7.8, p<0.05$ ). The effect of day, and the interactions of time and sex, day and sex, time and day, and time, sex, and day were all nonsignificant ( $p$ 's $>0.1)$. A Pearson correlation analysis revealed that there was no relationship between average total intake over the 7-day discrete trial procedure and the percent change from baseline (sucrose preference averaged across the initial four

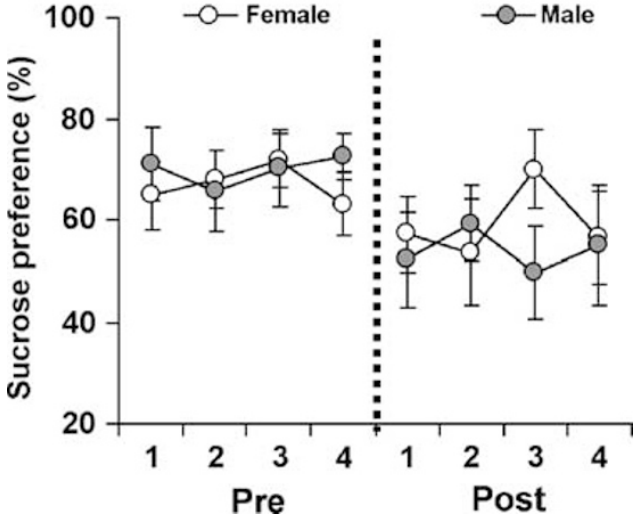

Figure 2 Decreased preference for sucrose following discrete trial selfadministration. Preference for sucrose over water is shown for the last four sucrose and water sessions prior to cocaine self-administration training (Pre) and the four sucrose and water session immediately after discrete trial self-administration (Post). Open circle represents data from female rats $(n=5)$ and filled circles represent data from male rats $(n=6)$.

sessions) to retest (sucrose preference averaged across the four retest sessions) for final ratios $\left(r^{2}=0.04\right)$. Thus, at retest, both males and females showed a significant decrease in preference for sucrose over water, and the change from baseline did not differ significantly between males and females.

Effect of Estrogen on Initial Cocaine Self-Administration under the Fixed Ratio and Progressive Ratio Schedules and during the Discrete Trial Sessions

The OVX $+\mathrm{EB}$ rats and OVX $+\mathrm{VEH}$ rats acquired cocaine self-administration in a similar number of days $(9.3 \pm 1.4$ and $7.7 \pm 0.8$ ) and obtained a similar number of infusions under the three sessions in which cocaine was available under a progressive ratio schedule $(17.0 \pm 1.6$ and $17.8 \pm 1.6)$ $(p$ 's $>0.1)$. However, similar to differences observed between males and females, OVX + EB rats self-administered more cocaine over the 7-day testing period under the 24-h discrete trial procedure than did OVX $+\mathrm{VEH}$ rats (see Figure 3a). A repeated measures ANOVA revealed a significant effect of group $\left(\mathrm{F}_{1,12}=16.5, p<0.01\right)$ and day $\left(\mathrm{F}_{6,72}=2.5, p<0.05\right)$, but no significant interaction of group by day $(p>0.1)$. Thus, estrogen replacement did not affect rates of acquisition or baseline final ratios reached under a progressive ratio schedule, but it did enhance daily levels of cocaine intake under extended access conditions. Responses on the activity lever were very low throughout the experiment, and there were no significant differences between the OVX $+\mathrm{EB}$ and OVX $+\mathrm{VEH}$ groups (data not shown).

Effect of Estrogen on Progressive Ratio Responding for Cocaine Prior to and Following Extended Cocaine Self-Administration

Figure $3 \mathrm{~b}$ shows data for the number of infusions and corresponding final ratios reached under the progressive ratio schedule for $\mathrm{OVX}+\mathrm{EB}$ and $\mathrm{OVX}+\mathrm{VEH}$ rats before and after cocaine self-administration under the discrete trial 

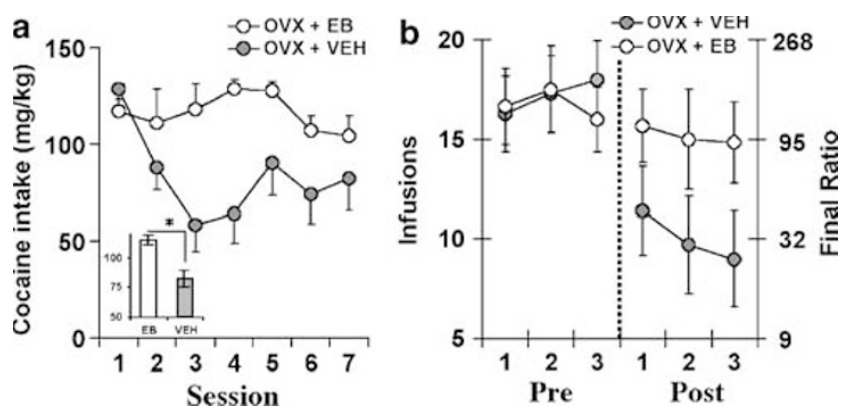

Figure 3 Effect of estrogen on responding under the discrete trial and progressive ratio cocaine self-administration procedures. (a) Data represent the mean $( \pm S E)$ daily cocaine intake for OVX rats with $(n=7$, open circles, OVX + EB) and without ( $n=7$, filled circles, OVX + VEH) estrogen replacement as a function of day under the discrete trial procedure. The inset shows the mean $( \pm S E)$ daily intake over the 7-day testing period for the OVX + EB (EB) and OVX + VEH (VEH) groups. Asterisks indicate a significant group difference $(p<0.05)$. (b) Mean $( \pm S E)$ number of infusions obtained and the corresponding final ratios reached under the progressive ratio schedule for the three baseline sessions before (Pre) and the three retest sessions after (Post) access to cocaine under the discrete trials procedure for $\mathrm{OVX}$ rats with $(n=7$, open circles, $\mathrm{OVX}+\mathrm{EB})$ and without $(n=7$, filled circles, OVX $+V E H)$ estrogen replacement.

procedure. The final ratios reached under the progressive ratio schedule were decreased from baseline levels in OVX + VEH rats, but not OVX + EB rats. Specifically, final ratios reached under the three progressive ratio retest sessions were decreased from those observed at the three baseline sessions by a mean of $45 \%( \pm 9 \%)$ in OVX $+\mathrm{VEH}$ rats compared to only $8 \%( \pm 6 \%)$ in OVX $+\mathrm{EB}$. The decrease observed in females was a persistent baseline change and values were constant across the three retest sessions. A repeated measures ANOVA revealed a significant main effect of time (Pre vs Post; $\mathrm{F}_{1,12}=25.3, p<0.001$ ) and a significant interaction of time by group $\left(\mathrm{F}_{1,12}=11.9\right.$, $p<0.01)$, and a nonsignificant effect of day $(p>0.1)$. Further analysis within the OVX $+\mathrm{EB}$ group and within the OVX + VEH group using separate repeated measures ANOVA revealed a significant effect of time for the OVX + VEH group $\left(\mathrm{F}_{1,6}=24.9, p<0.01\right)$, but not for the OVX $+\mathrm{EB}$ group $(p>0.1)$. Notably, a Pearson correlation analysis revealed that there was no relationship between total intake and the percent change from baseline to retest for final ratios $\left(r^{2}=0.02\right.$ OVX $\left.+\mathrm{EB}, r^{2}=0.07 \mathrm{OVX}+\mathrm{VEH}\right)$, indicating that the group difference in intake cannot account for the decreased breakpoints observed in the OVX $+\mathrm{VEH}$ group. Thus, following extended access cocaine selfadministration, estrogen blocked the decrease in motivation to obtain cocaine observed in OVX.

\section{Effect of Sex and Estrogen on Body Weights}

Despite high levels of intake under the 24-h access discrete trial procedure, rats maintained their health and only slight decreases in body weights were observed. Specifically, compared to the body weights observed prior to 24 -h access to cocaine under the discrete trial procedure, the body weights observed immediately following discrete trial cocaine self-administration were decreased by an average of $5.6 \%( \pm 1.3)$ in females and $3.5 \%( \pm 2.3)$ in males. A repeated measures ANOVA revealed a significant effect of group $\left(\mathrm{F}_{1,23}=86.5, p<0.0001\right)$ and time $\left(\mathrm{F}_{1,23}=9.56\right.$, $p<0.01)$, but no significant interaction of time and group $(p>0.1)$. Similar results were observed among the OVX groups. Specifically, compared to the body weights observed prior to 24-h access to cocaine under the discrete trial procedure, the body weights observed immediately following discrete trial cocaine self-administration were decreased by an average of $6.2 \%( \pm 3.8)$ in the OVX $+\mathrm{EB}$ group and $3.9 \%( \pm 2.0)$ in the OVX + VEH group. A repeated measures ANOVA revealed significant effects of group $\left(\mathrm{F}_{1,12}=9.2\right.$, $p<0.01)$ and time $\left(\mathrm{F}_{1,12}=4.7, p<0.05\right)$, but no significant interaction of time and group $(p>0.1)$. Thus, extended access to cocaine produced a similar degree of weight loss among males and females and OVX $+\mathrm{EB}$ and OVX $+\mathrm{VEH}$ rats. However, it should be noted that self-administration under these high access conditions resulted in signs of severe toxicity, particularly in females. Specifically, of 33 intact females tested to date under these high access conditions in our laboratory, four females died or showed rapid weight loss over the course of the 7-day 24-h access discrete trial procedure and two females showed signs of toxicity during the initial withdrawal period. In males, however, little evidence of toxicity has been observed, and out of 34 intact male rats that have been tested thus far, only one male has showed signs of toxicity. Similarly, signs of toxicity were rarely observed among OVX rats, and of the 15 rats tested, only one (OVX $+\mathrm{EB})$ showed signs of toxicity over the course of the 7-day 24-h access discrete trial procedure.

Slides obtained from vaginal swabs confirmed hormonal status from each of the OVX groups. Specifically, proestruslike cells predominated vaginal smears obtained from the $\mathrm{OVX}+\mathrm{EB}$ group, while metestrus/diestrus-like cell predominated vaginal smears obtained from the OVX $+\mathrm{VEH}$ group.

\section{DISCUSSION}

The goals of this investigation were to examine short-term motivational changes that occur in male and female rats following cocaine self-administration under extended access conditions and to determine the biological basis of observed sex differences. Results demonstrated that females selfadministered greater levels of cocaine under extended access conditions, and that following such access, females, but not males, showed a marked reduction in levels of responding for cocaine under the progressive ratio schedule. These findings indicate a sex difference after extended access to cocaine on subsequent motivation to obtain cocaine that appeared to be specific to cocaine in that both males and females showed a similar decrease in preference for sucrose under the conditions tested. Results from OVX females demonstrated that estrogen replacement produced an increase in cocaine self-administration under extended access conditions and blocked the decrease in motivation to obtain cocaine following such access. Taken together, these findings suggest that sex differences are found under extended cocaine access conditions, and that such access leads to sex- and hormone-specific changes in motivation to obtain cocaine. 


\section{Sex Differences}

Previous research in male rats has shown that preference for sucrose and other nondrug rewards decrease following chronic exposure to cocaine and other psychostimulants (Barr and Phillips, 1999, 2002; Markou and Koob, 1991; Kokkinidis and McCarter, 1990). This decrease has been interpreted as a reduced capacity for reward, or anhedonia. Here, we replicate these previous findings and extend them to include a decrease in preference for sucrose in both male and female rats and a decrease in motivation to obtain cocaine in female rats.

We have recently reported that female rats self-administer greater levels of cocaine under the discrete trial cocaine selfadministration procedure than do male rats, and that following such access, they show greater increases in motivation to obtain cocaine when tested after an extended abstinence period (Lynch and Taylor, 2004). In the current study, we tested the hypothesis that such access conditions might produce sex differences in more immediate motivational changes. Here, we found that motivation to obtain cocaine was decreased in females tested shortly after the discrete trial procedure in contrast to the later time points (ie 10 days after discrete trial self-administration) where females showed increased motivation to obtain cocaine (Lynch and Taylor, 2004). The sex difference in motivation appeared to be specific to cocaine because males and females showed a similar decrease in preference for sucrose following extended access to cocaine. Additionally, the sex difference in motivation to obtain cocaine cannot be easily attributed to a sex difference in drug intake under extended access conditions because there was no significant correlation between total intake under the discrete trial procedure and a change in motivation to obtain cocaine as measured by progressive ratio responding. Together, these findings suggest that motivation to obtain cocaine varies over the abstinence period and that there is a dissociation between sex and the short- $v s$ long-term alteration in motivation to use cocaine.

In contrast to previous research from our group and others demonstrating sex differences in rates of acquisition of cocaine self-administration and levels of responding under the progressive ratio schedule (Roberts et al, 1989; Carroll et al, 2002; Lynch and Carroll, 1999; Hu et al, 2004), no sex differences were observed under either the fixed ratio or the progressive ratio schedule prior to $24-\mathrm{h}$ access to cocaine under the discrete trial procedure. A likely explanation for these discrepancies is that the high cocaine dose tested in the present experiment may have obscured sex differences. Specifically, previous work has shown that under high-dose conditions, like those used in the present experiment, males and females do not differ in rates of acquisition (Haney et al, 1995; Roberts et al, 1989; Lynch et al, 2001; Lynch and Taylor, 2004; Caine et al, 2004) or in progressive ratio responding for cocaine (Lynch and Taylor, 2004). However, when males and females are compared under low cocaine dose conditions, rates of acquisition and levels of responding under the progressive ratio schedule are highly variable and sex differences are revealed with females acquiring at a much faster rate and reaching much higher final ratios than do males (Roberts et al, 1989; Carroll et al, 2002; Lynch and Carroll, 1999; Hu et al, 2004).
Importantly, under these extended access conditions the same dose of cocaine maintained higher levels of intake in females compared to males, suggesting that although this dose of cocaine is considered to be relatively high under fixed ratio and progressive ratio testing conditions, it may be at a threshold level for the production of sustained high levels of intake for male, but not female rats. In fact, the levels of intake observed in female rats in the present experiment and in previous work (Lynch and Taylor, 2004; Lynch et al, 2004) are similar to those observed in male rats tested under higher cocaine access conditions (eg five trials/ $\mathrm{h}$ with $1.5 \mathrm{mg} / \mathrm{kg} / \mathrm{inj}$ cocaine; Lynch and Roberts, 2004; Roberts et al, 2002).

\section{Effects of Estrogen}

Following extended access to cocaine, like intact females, OVX females without estrogen replacement showed a decrease in motivation to obtain cocaine, whereas, like males, OVX females with estrogen replacement did not show a change from baseline in motivation to obtain cocaine. These findings are somewhat surprising in that OVX rats with estrogen are typically similar to intact females on cocaine self-administration behavior, while OVX rats without estrogen are typically behaviorally similar to males. One possible explanation is that at retest, intact females had very low levels of circulating estrogen. Specifically, chronic cocaine administration has been reported to disrupt the estrous cycle producing prolonged periods associated with low levels of estrogen (ie metestrus/ diestrus) and very few periods associated with high levels of estrogen (ie proestrus and estrus). Additionally, previous research has shown that while acutely administered cocaine results in increased estradiol levels (Mello and Mendelson, 2002), chronically administered cocaine results in decreased levels (Thyer et al, 2001). However, it should be noted that even when estrogen levels are low (ie during the metestrus/ diestrus phase), females have been reported to respond at higher or comparable levels for cocaine than do males under both the progressive ratio and fixed ratio schedules (Roberts et al, 1989; Lynch et al, 2002). Thus, even if the intact females tested in the present study had low levels of estrogen as a consequence of exposure to cocaine, this explanation is unlikely to fully account for the sex difference in subsequent motivation to obtain cocaine. However, it is possible that such depletion of estrogen combined with extended access cocaine self-administration permits a dysregulation of the reward systems that is manifest as a reduction in motivation to obtain cocaine. Alternatively, it is possible that hormones other than estrogen (eg progesterone) may be responsible for the marked decrease in motivation to obtain cocaine observed in intact females. Future studies are necessary to investigate the relationship between levels of circulating ovarian hormones and motivation to obtain cocaine.

Consistent with previous research under low-dose acquisition testing conditions (Lynch and Carroll, 1999; Hu et al, 2004), daily levels of intake under these high-dose extended access conditions were reduced by ovariectomy and restored by estrogen replacement. Specifically, the levels of cocaine intake obtained by estrogen-replaced $(\approx 115 \mathrm{mg} /$ $\mathrm{kg})$ and vehicle-treated $(\approx 83 \mathrm{mg} / \mathrm{kg})$ OVX rats were similar 
to those obtained by intact female $(\approx 105 \mathrm{mg} / \mathrm{kg})$ and male rats $(\approx 90 \mathrm{mg} / \mathrm{kg})$, respectively. Taken together, these results demonstrate that estrogen modulates levels of cocaine self-administration under high access conditions in females and that such effects may be related to the observed sex differences. The effect of estrogen on cocaine self-administration in females is likely to occur through interactions with dopamine. Specifically, the mesolimbic dopamine system is believed to mediate the reinforcing effects of cocaine (Koob and Nestler, 1997). Previous research has shown that dopamine release and reuptake in this area fluctuate over the estrous cycle (Shimizu and Bray, 1993), and that in OVX females, EB modifies dopamine activity (Shimizu and Bray, 1993) and alters stimulantinduced dopamine release in the striatum (Peris et al, 1991; Becker, 1990).

We have recently reported sex differences in diurnal patterns and levels of cocaine self-administration under these extended access conditions (Lynch and Taylor, 2004) and unpublished results from our laboratory have revealed similar effects with estrogen replacement. An examination of the conditions under which estrogen, and other ovarian hormones, including progesterone, modulate levels and patterns of cocaine self-administration under high access conditions is the focus of ongoing studies.

\section{CONCLUSIONS}

Data from the clinical literature indicate sex differences in the effects of chronic cocaine on subsequent motivation to use the drug. For example, women report shorter cocaine abstinence periods than do men (Kosten et al, 1996), and they are more likely than men to attribute relapse to cocaine use depression or negative affect (McKay et al, 1996), suggesting that withdrawal from drugs may be associated with greater negative affect in women than in men. Evidence from studies with laboratory animals also demonstrate that females are more sensitive to the reinforcing and behavioral activating effects of cocaine, and show an increase in vulnerability during the acquisition and relapse phases using drug self-administration paradigms (Carroll et al, 2004; Lynch et al, 2002). The results from this study showed sex differences in levels of cocaine self-administration under extended access conditions and in the effects of such access on subsequent motivation to obtain cocaine. Results from OVX rats show that estrogen modulates cocaine selfadministration behavior under high access conditions in females, and they suggest that such effects may be related to sex differences in vulnerability to cocaine. Studies comparing males and females after cocaine self-administration under extended access conditions on subsequent behavioral, motivational, and neurochemical measures, and their ability to predict vulnerability to self-administration may provide important information regarding mechanisms underlying sex differences in addiction.

\section{ACKNOWLEDGEMENTS}

We are grateful to Victoria Stewart and Lauren Mangini for their technical assistance. We would like to acknowledge the Yale Interdisciplinary Women's Health Research Scholar
Program on Women and Drug Abuse, NIH Office of Research on Women's Health. This work was supported by grants from NIDA (DA11717, DA016556 and DA114038).

\section{REFERENCES}

Ahmed SH, Kenny PJ, Koob GF, Markou A (2002). Neurobiological evidence for hedonic allostasis associated with escalating cocaine use. Nat Neurosci 5: 625-626.

American Psychiatric Association (1994). Diagnostic and Statistical Manual of Mental Disorders, 4 th edn. American Psychiatric Press: Washington, DC.

Barr AM, Phillips AG (1999). Withdrawal following repeated exposure to $\mathrm{D}$-amphetamine decreases responding for a sucrose solution as measured by a progressive ratio schedule of reinforcement. Psychopharmacology 141: 99-106.

Barr AM, Phillips AG (2002). Increased successive negative contrast in rats withdrawn from an escalating-dose schedule of D-amphetamine. Pharmacol Biochem Behav 71: 293-299.

Becker JB (1990). Estrogen rapidly potentiates amphetamineinduced striatal dopamine release and rotational behavior during microdialysis. Neurosci Lett 118: 169-171.

Becker JB (1999). Gender differences in dopaminergic function in striatum and nucleus accumbens. Pharmacol Biochem Behav 64: 803-812.

Caine SB, Bowen CA, Yu G, Zuzga D, Negus SS, Mello NK (2004). Effect of gonadectomy and gonadal hormone replacement on cocaine self-administration in female and male rats. Neuropsychopharmacology 29: 929-942.

Carroll ME, Lynch WJ, Roth ME, Morgan AD, Cosgrove KP (2004). Sex and estrogen influence drug abuse. Trends Pharmacol Sci 25: 273-279.

Carroll ME, Morgan AD, Campbell UC, Lynch WJ, Dess NK (2002). Influence of estrogen in the acquisition of intravenously selfadministration in rats selectively bred for differential saccharin intake: phenotype and sex differences. Psychopharmacology 161: 304-313.

Fitch TE, Roberts DCS (1993). The effects of dose and access restrictions on the periodicity of cocaine self-administration in the rat. Drug Alcohol Depend 33: 119-128.

Gawin FH, Kleber HD (1986). Abstinence symptomatology and psychiatric diagnosis in cocaine abusers. Clinical observations. Arch Gen Psychiatry 43: 107-113.

Grimm JW, Hope BT, Wise RA, Shaham Y (2001). Neuroadaptation. Incubation of cocaine craving after withdrawal. Nature 412: 141-142.

Haney M, Maccari S, Le Moal M, Simon H, Piazza PV (1995). Social stress increases the acquisition of cocaine self-administration in male and female rats. Brain Res 698: 46-52.

$\mathrm{Hu}$ M, Crombag HS, Robinson TE, Becker JB (2004). Biological basis of sex differences in the propensity to self-administer cocaine. Neuropsychopharmacology 29: 81-85.

Jentsch JD, Taylor JR (1999). Impulsivity resulting from frontostriatal dysfunction in drug abuse: implications for the control of behavior by reward-related stimuli. Psychopharmacology 146: 373-390.

Kampman KM, Volpicelli JR, Mulvaney F, Rukstalis M, Alterman AI, Pettinati $\mathrm{H}$ et al (2002). Cocaine withdrawal severity and urine toxicology results from treatment entry predict outcome in medication trials for cocaine dependence. Addict Behav 27: 251-260.

King TS, Canez MS, Gaskill S, Javors MA, Schenken RS (1993). Chronic cocaine disruption of estrous cyclicity in the rat: dosedependent effects. J Pharmacol Exp Ther 264: 29-34.

Kokkinidis L, McCarter BD (1990). Postcocaine depression and sensitization of brain-stimulation reward: analysis of reinforcement and performance effects. Pharmacol Biochem Behav 36: 463-471. 
Koob GF, Ahmed SH, Boutrel B, Chen SA, Kenny PJ, Markou A et al (2004). Neurobiological mechanisms in the transition from drug use to drug dependence. Neurosci Biobehav Rev 27: 739-749.

Koob GF, Nestler EJ (1997). The neurobiology of drug addiction. J Neuropsychiatry Clin Neurosci 9: 482-497.

Kosten TR, Kosten TA, McDougle CJ, Hameedi FA, McCance EF, Rosen MI et al (1996). Gender differences in response to intranasal cocaine administration to humans. Biol Psychiatry 39: 147-148.

Lynch WJ, Arizzi MN, Carroll ME (2000). Effects of sex and the estrous cycle on regulation of intravenously self-administered cocaine in rats. Psychopharmacology 152: 132-139.

Lynch WJ, Carroll ME (1999). Sex differences in the acquisition of intravenously self-administered cocaine and heroin in rats. Psychopharmacology 144: 77-82.

Lynch WJ, Mangini LD, Taylor JR (2004). Neonatal isolation stress potentiates cocaine seeking behavior in adult male and female rats. Neuropsychopharmacology (in press).

Lynch WJ, Roberts DCS (2004). Effects of cocaine self-administration on food-reinforced responding using a discrete trial procedure in rats. Neuropsychopharmacology 29: 669-675.

Lynch WJ, Roth ME, Carroll ME (2002). Biological basis of sex differences in drug abuse: preclinical and clinical studies. Psychopharmacology 164: 121-137.

Lynch WJ, Roth ME, Mickelberg JL, Carroll ME (2001). Role of estrogen in the acquisition of intravenously self-administered cocaine in female rats. Pharmacol Biochem Behav 68: 641-646.

Lynch WJ, Taylor JR (2004). Sex differences in the behavioral effects of 24-h/day access to cocaine under a discrete trial procedure. Neuropsychopharmacology 29: 943-951.

Markou A, Koob GF (1991). Postcocaine anhedonia. An animal model of cocaine withdrawal. Neuropsychopharmacology 4: 17-26.

McKay JR, Rutherford MJ, Cacciola JS, Kabasakalian-McKay R, Alterman AI (1996). Gender differences in the relapse experiences of cocaine patients. J Nerv Ment Dis 184: 616-622.

Mello NK, Mendelson JH (2002). Cocaine, hormones, and behavior: clinical and preclinical studies. Hormones Brain Behav 5: 665-745.

Morgan D, Brebner K, Lynch WJ, Roberts DCS (2002). Increases in the reinforcing efficacy of cocaine after particular histories of reinforcement. Behav Pharmacol 13: 389-396.
Mulvaney FD, Alterman AI, Boardman CR, Kampman K (1999). Cocaine abstinence symptomatology and treatment attrition. J Subst Abuse Treat 16: 129-135.

Peris J, Decambre N, Coleman-Hardee ML, Simpkins JW (1991). Estradiol enhances behavioral sensitization to cocaine and amphetamine-stimulated striatal $\left[{ }^{3} \mathrm{H}\right]$ dopamine release. Brain Res 566: 255-264.

Pfaus JG, Pfaff DW (1992). Mu-, delta-, and kappa-opioid receptor agonists selectively modulate sexual behaviors in the female rat: differential dependence on progesterone. Horm Behav 26: 457-473.

Richardson NR, Roberts DCS (1996). Progressive ratio schedules in drug self-administration studies in rats: a method to evaluate reinforcing efficacy. J Neurosci Methods 66: 1-11.

Roberts DCS, Bennett SAL, Vickers GJ (1989). The estrous cycle affects cocaine self-administration on a progressive ratio schedule in rats. Psychopharmacology 98: 408-411.

Roberts DCS, Brebner K, Vincler M, Lynch WJ (2002). Patterns of cocaine self-administration in rats produced by various access conditions under a discrete trials procedure. Drug Alcohol Depend 67: 291-299.

Robinson TE, Berridge KC (2001). Incentive-sensitization and addiction. Addiction 96: 103-114.

Shimizu H, Bray GA (1993). Effects of castration, estrogen replacement and estrus cycle on monoamine metabolism in the nucleus accumbens, measured by microdialysis. Brain Res 621: 200-206.

Sofuoglu M, Dudish-Poulsen S, Nelson D, Pentel PR, Hatsukami DK (1999). Sex and menstrual cycle differences in the subjective effects from smoked cocaine in humans. Exp Clin Psychopharmacol 7: 274-283.

Stock HS, Ford K, Wilson MA (2000). Gender and gonadal hormone effects in the olfactory bulbectomy animal model of depression. Pharmacol Biochem Behav 67: 183-191.

Tarttelin MF, Gorski RA (1973). The effects of ovarian steroids on food and water intake and body weight in the female rat. Acta Endocrinol (Copenh) 72: 551-568.

Thyer AC, King TS, Moreno AC, Eddy CA, Siler-Khodr TM, Schenken RS et al (2001). Cocaine impairs ovarian response to exogenous gonadotropins in nonhuman primates. J Soc Gynecol Invest 8: 358-362. 